\title{
Short-term survival and safety of apatinib combined with oxaliplatin and S-1 in the conversion therapy of unresectable gastric cancer
}

Zaisheng Ye ${ }^{1 \dagger}$, Yi Zeng ${ }^{1 \dagger}$, Shenghong Wei ${ }^{1}$, Yi Wang ${ }^{1}$, Zhitao Lin ${ }^{1}$, Shu Chen ${ }^{1}$, Zhiwei Wang ${ }^{1}$, Shanshan Chen ${ }^{2}$ and Luchuan Chen $^{1 *}$

\begin{abstract}
Background: We conducted a single-arm phase II trial to investigate the short-term efficacy and safety of apatinib combined with oxaliplatin and S-1 in the treatment of unresectable gastric cancer.

Patients and methods: Previously untreated patients with unresectable HER-2-negative advanced gastric cancer were selected. All the patients received six cycles of S-1 and oxaliplatin and five cycles of apatinib, which were administered at intervals of three weeks. The surgery was performed after six cycles of drug treatment. The primary endpoints were radical resection (RO) rate and safety. This study was registered with the China Trial Register, number ChiCTR-ONC-17010430 (01/12/2016-01/12/2022).

Results: A total of 39 patients were enrolled. Efficacy evaluation was feasible for 37 patients. One patient achieved complete response $(C R, 2.7 \%), 26$ patients achieved partial response (PR, 70.3\%), three patients had stable disease $(\mathrm{SD}, 8.1 \%)$ and seven patients had progressive disease (PD, 18.9\%). The objective response rate (ORR) was $73.0 \%$ and the disease control rate (DCR) was $81.1 \% .22$ patients underwent surgery, among which 14 patients underwent radical resection (R0), with a R0 resection rate of 63.6\%. The 1-year survival rate of the surgical group (22 patients) was $71.1 \%$ and the 2-year survival rate was $41.1 \%$. The median survival time was 21 months. The incidence of adverse events (AEs) was 100\%. Leucopenia (65.3\%) and granulocytopenia (69.2\%) were the most common hematological AEs. The most common non-hematological AEs were fatigue (51.3\%) and oral mucositis (35.9\%).

Conclusion: Apatinib combined with oxaliplatin and S-1 showed good short-term survival and acceptable safety in the conversion therapy of unresectable gastric cancer.
\end{abstract}

Keywords: Unresectable gastric cancer, Conversion therapy, Apatinib, Efficacy, Safety

\footnotetext{
* Correspondence: luchuanchen@sina.cn

${ }^{\dagger}$ Zaisheng Ye and Yi Zeng contributed equally to this work.

${ }^{1}$ Department of Gastrointestinal Surgical Oncology, Fujian Cancer Hospital \&

Fujian Medical University Cancer Hospital, Fuzhou 350014, Fujian Province,

China
}

Full list of author information is available at the end of the article

\section{$\triangle B M C$}

(c) The Author(s). 2021 Open Access This article is licensed under a Creative Commons Attribution 4.0 International License, which permits use, sharing, adaptation, distribution and reproduction in any medium or format, as long as you give appropriate credit to the original author(s) and the source, provide a link to the Creative Commons licence, and indicate if changes were made. The images or other third party material in this article are included in the article's Creative Commons licence, unless indicated otherwise in a credit line to the material. If material is not included in the article's Creative Commons licence and your intended use is not permitted by statutory regulation or exceeds the permitted use, you will need to obtain permission directly from the copyright holder. To view a copy of this licence, visit http://creativecommons.org/licenses/by/4.0/. The Creative Commons Public Domain Dedication waiver (http://creativecommons.org/publicdomain/zero/1.0/) applies to the data made available in this article, unless otherwise stated in a credit line to the data. 


\section{Background}

In China, unresectable gastric cancer accounts for $10 \%$ of the total number of gastric cancer cases [1]. At present, palliative chemotherapy is the main treatment option. The median survival time is 5-12 months, and the 5-year survival rate is about 9.4\% [2]. In unresectable gastric cancer, the primary focus of gastric cancer infiltrates into the extraserous or surrounding tissues and organs, and distant metastasis occurs, such as paraaortic, liver, peritoneum, etc. Therefore, radical resection is difficult from the perspective of surgical technology and oncology. The conversion therapy provides a new therapeutic option in clinical practice. Through multidisciplinary treatment (MDT) mode, the patients are given reasonable chemotherapy, radiotherapy and targeted treatment, so that the initial non-resectable tumor can be transformed into resectable tumor, in order to prolong the survival outcome and improve the quality of life of the patients. More and more literatures reported that patients who responded to chemotherapy or targeted treatment and then underwent gastrectomy (stage IV gastric cancer) had longer survival time [3-6].

The combination of oxaliplatin and S-1 (SOX) has been widely used in neoadjuvant and postoperative chemotherapy of gastric cancer [7-9]. Apatinib, a new small molecular inhibitor of vascular endothelial growth factor receptor, which is a third-line treatment option for advanced gastric cancer, has achieved reliable results [10] The combination of SOX and apatinib has also been applied in neoadjuvant chemotherapy of advanced gastric cancer [11]. However, there are few reports on its application in conversion therapy, especially on the survival rate. Therefore, this study examined the short-term survival effect and safety of apatinib combined with oxaliplatin and S-1 in the treatment of unresectable gastric cancer.

\section{Patients and methods Patients}

All the patients were confirmed to be adenocarcinoma by biopsy under gastroscopy, and HER-2 negative by immunohistochemistry. Inclusion criteria were: age 18-70 years; confirmed by pathology as gastric adenocarcinoma, and met one of the following non-resectable conditions: peritoneal metastasis, liver metastases, Krukenberg tumor, distant lymph node metastasis, N3, extensive or bulky lymph nodes, local progression; CT/MRI before operation or color ultrasound, PET-CT, if necessary, laparoscopic exploration to clearly diagnose the abovementioned staging of gastric cancer; diagnosed patients who had not received prior radiotherapy, chemotherapy, targeted treatment or immunotherapy; ECOG 0-1; expected survival time $\geq 3$ months; no severe dysfunction of heart, lung and liver; no jaundice and digestive tract obstruction; no acute infection; normal function of main organs; no pregnancy; without involving other clinical trial.

\section{Treatment}

The patients were treated with apatinib, oxaliplatin and S-1. Oxaliplatin was administered every 3 weeks at 130 $\mathrm{mg} / \mathrm{m} 2$ intravenously on day 1 according to the body surface area; Apatinib was given orally (500 mg/day) for continuous 5 cycles, followed by a one-cycle rest. S-1: according to the body surface area, the dosage $\left(<1.25 \mathrm{~m}^{2}\right.$, $40 \mathrm{mg}$, bid; $1.25-1.50 \mathrm{~m}^{2}, 50 \mathrm{mg}$, bid; > $1.50 \mathrm{~m}^{2}, 60 \mathrm{mg}$, bid, P. O, bid, d1-d14) was given orally from the first day of chemotherapy for 2 weeks, followed by a 1-week break. The short-term efficacy was evaluated every two cycles. The operation was performed when the requirements of surgical resection were met. All the patients received six cycles of conversion therapy (the last cycle stopped using apatinib). According to RECIST 1.1, complete response $(\mathrm{CR})$ and partial response (PR) were regarded as objective remission rate (ORR), while $C R$, $\mathrm{PR}$ and stable disease (SD) were regarded as disease control rate (DCR). The adverse reactions were divided into 0-IV degrees according to the National Cancer Institute Common Terminology Criteria for Adverse Events (NCI-CTCAE 4.0).

Three weeks after the therapy, the doctors performed conventional laparotomy or laparoscopic exploration, and decided the operation plan based on the situation. D2 radical resection was the first choice. Palliative resection and/or short circuit operation of digestive tract were selected if radical resection of local cancer was not feasible. In the surgical group, the original treatment was continued for two cycles after operation, and then S-1 and apatinib were taken orally for six months. The pathological response of the surgical group was evaluated in accordance with the 14th edition of Japanese gastric cancer treatment protocol, in which the pathological response rate (PRR) refers to the survival of tumor cells in less than two-third of the tumor area (IB or above). Tumor staging (cTNM and ypTNM) was determined according to the AJCC seventh edition.

Each patient was followed-up once every three months after adjuvant treatment until death. The follow-up methods included telephone, short message, outpatient visit, etc. The follow-up items included survival, endoscopy, abdominal enhanced CT, tumor markers and routine laboratory tests.

\section{Statistical analysis}

According to the historical data of SOX conversion therapy, R0 resection rate (H0 value): 36\% [7-9]; Expected SOX+Apaninb conversion therapy, R0 resection rate $(\mathrm{H} 1)$ : $60 \%$; bilateral $\alpha=0.05, \beta=0.2$. The calculated 
Table 1 Baseline characteristics of the patients

\begin{tabular}{ll}
\hline Variable & Patients ( $\mathbf{N = 3 9 )}$ \\
\hline Age (years), median (range) & $58(30-68)$ \\
Gender, N (\%) & $19(48.7)$ \\
Male & $20(51.3)$ \\
Female & \\
ECOG performance status, N (\%) & $12(30.8)$ \\
0 & $27(69.2)$ \\
1 & \\
Non resectable factors, N (\%) & $2(5.1)$ \\
N3 lymph node metastasis & $4(10.3)$ \\
Distant lymphatic metastasis & $4(10.3)$ \\
Invasion of peripheral organs (T4b) & $2(5.1)$ \\
Liver metastasis & $27(69.2)$ \\
Peritoneal carcinomatosis & \\
Pathological type, N (\%) & $5(12.8)$ \\
Moderately differentiated adenocarcinoma & $17(43.6)$ \\
Poorly differentiated adenocarcinoma & $3(7.7)$ \\
Mucinous adenocarcinoma & $14(35.9)$ \\
Signet ring cell carcinoma & \\
Tumor site, N (\%) & $11(28.2)$ \\
Antrum gastric angle & $11(28.2)$ \\
Gastric body & $10(25.6)$ \\
Fundus cardia & $7(17.9)$ \\
Diffuse whole stomach &
\end{tabular}

sample size is: $n=32$, considering the shedding rate of $20 \%$, a total of 40 patients need to be included in the group. SPSS 19.0 statistical software was used for statistical analysis. All measurement data with normal distribution are expressed in $(x \pm s)$. The mean value of the two groups was compared by the Student's $t$-test, and the count data were tested by the $\chi^{2}$ test or the Fisher exact probability method. Kaplan-Meier method was used for survival analysis, and the survival rate was compared by Log-rank test. A $p$-value $<0.05$ indicated statistically significant difference.

\section{Results}

\section{Patient characteristics}

Between December 2016 and May 2019, 39 patients with advanced unresectable gastric cancer were selected. The average age of the patients was 58 years. The pathological types were signet ring cell carcinoma and poorly differentiated adenocarcinoma. The major nonresectable factors were peritoneal metastasis, N3, liver metastasis and local progression, as shown in Table 1.

\section{Efficacy evaluation}

No patient was lost to follow-up. The follow-up time was 12-40 months up to April 2020. After pre-operative treatment, 37 patients could be evaluated (one case was retreated due to IV myelosuppression, and one case was dropped-out due to acute upper gastrointestinal hemorrhage and perforation). Among the 37 patients, one patient achieved CR (2.7\%), 26 achieved PR (70.3\%), three had SD (8.1\%) and seven had PD (18.9\%). The ORR was $73.0 \%$, and DCR was $81.1 \%$.

All patients without PD were advised to undergo laparoscopic exploration again and receive surgical treatment as possible. 9 patients with PR were not willing to accept surgery due to personal reasons (Table 2).Finally, a total of 22 patients $(59.6 \%)$ underwent surgery after laparoscopic exploration confirming the curative efficacy, of which 14 underwent radical resection (R0). Intraoperative R0 resection refers to the removal of all visible metastases on the basis of open standard D2 radical surgery, and ensure sufficient margin. The rate of R0 resection was $63.6 \%$ (Table 3). PET-CT changes of one case with liver metastasis alone before and after the

Table 2 Characteristics of surgical group and non surgical group

\begin{tabular}{lll}
\hline Variable & $\begin{array}{l}\text { Surgical group, } \\
\text { N (\%) }\end{array}$ & $\begin{array}{c}\text { Non Surgical group, } \\
\text { N (\%) }\end{array}$ \\
\hline Efficacy evaluation & & $0(0)$ \\
CR & $1(4.5)$ & $9(42.9)$ \\
PR & $18(81.8)$ & $0(0)$ \\
SD & $3(13.7)$ & $7(57.1)$ \\
PD & $0(0)$ & $0(0)$ \\
Unresectable factors & & $2(12.4)$ \\
N3 lymph node metastasis & $2(9.1)$ & $1(6.3)$ \\
Distant lymphatic metastasis & $2(9.1)$ & $1(6.3)$ \\
Invasion of peripheral organs (T4b) & $3(13.7)$ & $12(75.0)$ \\
Liver metastasis & $1(4.5)$ & $14(63.6)$ \\
Peritoneal carcinomatosis & & \\
\hline
\end{tabular}


Table 3 Total efficacy evaluation

\begin{tabular}{ll}
\hline Variable & Patients, N (\%) \\
\hline CR & $1(2.7)$ \\
PR & $26(70.3)$ \\
SD & $3(8.1)$ \\
PD & $7(18.9)$ \\
ORR & $27(73.0)$ \\
DCR & $30(81.1)$ \\
Surgical conversion & $22(59.5)$ \\
R0 resection & $14(63.6)$ \\
\hline
\end{tabular}

conversion treatment are shown in Fig. 1. The images of exploration of another case of peritoneal metastasis before and after the conversion treatment are shown in Fig. 2.

\section{Safety and adverse events}

The incidence of adverse events (AEs) was 100\%. Leucopenia (65.3\%), granulocytopenia (69.2\%) and thrombocytopenia (15.4\%) were the most common hematological AEs. The most common nonhematological AEs included fatigue (51.3\%), oral mucositis $(35.9 \%)$, and hypertension (25.6\%). No serious surgical complications were observed (Table 4).

\section{Postoperative pathological examination}

In terms of postoperative pathological response, seven cases were Grade IA (31.8\%), nine cases were Grade IB (40.9\%), five cases were Grade II (22.7\%), one case was Grade III (4.5\%), and the pathological response rate (PRR) was 68.2\% (Table 5).

\section{Short-term survival efficacy}

The 1-year survival rate of the surgical group (22 patients) was $71.1 \%$, the 2 -year survival rate was $41.1 \%$, and the median survival time was 21 months. The 1 -year survival rate of the non-surgical group (15 patients) was $61.4 \%$, the 2 -year survival rate was $24.5 \%$, and the median survival time was 12 months. There was a significant difference between the two groups in short-term survival $(p=0.026)$ (Fig. 3a).

Among the 27 patients with peritoneal metastasis, the 1 -year survival rate was $77.5 \%$, the 2 -year survival rate was $34.0 \%$, and the median survival time was 18 months. Among the 12 patients without peritoneal metastasis, the 1-year survival rate was $75.0 \%$, the 2 -year survival rate was $40.0 \%$, and the median survival time was 17 months. There was no significant difference between the two groups in short-term survival ( $p=0.084)$ (Fig. 3b).

In the surgical group, 14 cases received R0 resection. Among the 14 patients, 4 patients had recurrence and metastasis during follow-up, 2 patients had peritoneal metastasis, 1 patient had liver metastasis, and 1 patient had bilateral ovarian metastasis. The 1-year survival rate was $88.9 \%$, the 2 -year survival rate was $74.1 \%$. 8 cases received R1/R2 resection. The 1-year survival rate was $62.5 \%$, the 2 -year survival rate was $0 \%$. There was a significant difference between the two groups in short-term survival $(p=0.049)$, but there was no significant difference between the non-surgical group and the R1/R2 resection group ( $p=0.186$ ) (Fig. 3c).

In the surgical group, seven cases were diagnosed with pathological grade 0 -Ia. The 1 -year survival rate was $30.0 \%$. The 2 -year survival rate was $0 \%$. The median survival time was 12 months; 15 cases were diagnosed with grade Ib-III. The 1-year survival rate was $84.6 \%$, 2 -year survival rate was $47.0 \%$. The median survival time was

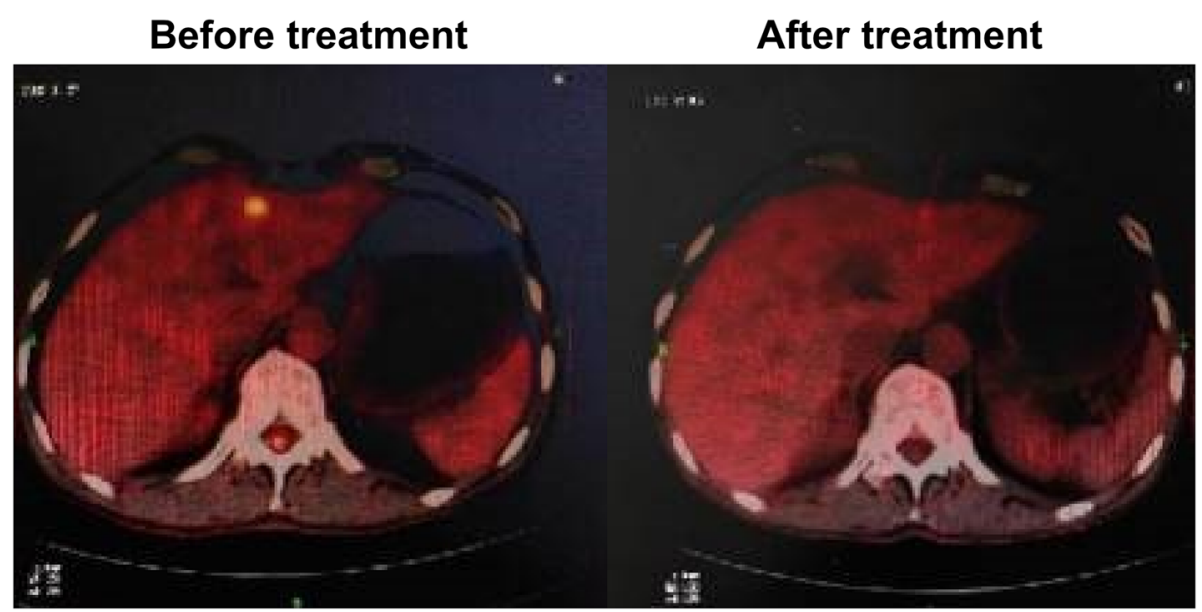

Fig. 1 Case 1 (liver metastasis alone) 


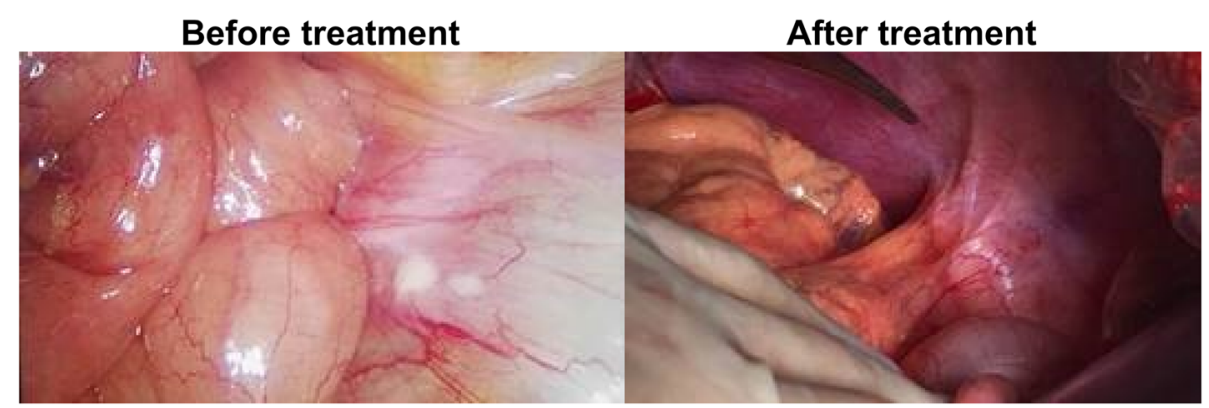

Fig. 2 Case 2 (Peritoneal metastasis)

21 months. There was significant difference between the two groups in short-term survival $(p=0.031)$ (Fig. 3d).

\section{Discussion}

This was a single arm, phase II clinical study. The main purpose of this study was to evaluate the conversion effect of SOX regimen combined with apatinib in the treatment of unresectable gastric cancer. The primary endpoints were R0 resection rate and safety. The secondary outcome measures were the objective response rate (ORR) and overall survival rate (OSR) of the whole group. Although the follow-up time was $<5$ years, we believe that the analysis of short-term survival effect would help to identify the improvement of prognosis of the combined regimen to a certain extent.

In terms of safety, given that the pre-operative course of conversion treatment was up to six cycles,and oral maintenance therapy was needed for half a year after operation. Only three patients $(8.1 \%)$ in this study had grades III and IV adverse reactions. One patient was withdrew due to grade IV severe thrombocytopenia, one patient withdrew due to emergency operation for upper

Table 4 Incidence of adverse reactions

\begin{tabular}{lll}
\hline Variable & \multicolumn{2}{l}{ Patients (N = 39) } \\
\cline { 2 - 3 } & Any grade, N (\%) & Grade 3 or 4, N (\%) \\
\hline Leukopenia & $27(73.0)$ & $0(0)$ \\
Granulocytopenia & $29(78.4)$ & $0(0)$ \\
Thrombocytopenia & $6(16.2)$ & $1(2.7)$ \\
Elevated transaminase & $9(24.3)$ & $0(0)$ \\
Hand-foot syndrome & $9(24.3)$ & $0(0)$ \\
Stomatitis & $14(37.8)$ & $0(0)$ \\
Fatigue & $20(54.1)$ & $0(0)$ \\
Proteinuria & $4(10.8)$ & $0(0)$ \\
Gastrointestinal hemorrhage & $2(5.4)$ & $1(2.7)$ \\
Hypertension & $10(27.0)$ & $1(2.7)$ \\
Neurotoxicity & $10(27.0)$ & $0(0)$ \\
Gastrointestinal perforation & $1(2.7)$ & $1(2.7)$ \\
\hline
\end{tabular}

gastrointestinal hemorrhage and perforation. In the case of repeated grade III hypertension and poor antihypertensive effect, one patient could control the blood pressure level within the normal range after reducing the dosage of apatinib to $250 \mathrm{mg}$ per day, and then received surgical treatment. Apatinib is an anti-angiogenic drug that may induce gastrointestinal perforation and bleeding, which is most likely to cause treatment interruption. However, in this study, except for one patient with upper gastrointestinal hemorrhage and perforation, only one patient had black stool symptoms in the sixth cycle of pre-operative treatment. At that time, the patient had stopped using apatinib for 2 weeks, and all S-1 oral drugs had been consumed, so after fasting, omeprazole and somatostatin treatment, the bleeding stopped quickly. And then the patient successfully underwent R0 resection. Therefore, the combined therapy does not highlight the side effects of apatinib on anti-angiogenesis. The main AEs were myelosuppression, oral mucositis and asthenia. Myelosuppression was mainly leukopenia and granulocytopenia, mostly grade I and II, which were tolerable. Oral mucositis was also mild, and could be relieved after vitamin $\mathrm{C}$ treatment and local symptomatic treatment. The incidence of adverse reactions was not higher than that of oxaliplatin and S-1 alone $[7,9]$. The incidence of asthenia, hypertension, albuminuria and hand foot syndrome was higher than that of oxaliplatin and S-1 alone reported in the literature [7, 9], but could be easily controlled due to the mild degree of I and II, which did not affect its clinical use. Sym et al. [12]. used docetaxel + capecitabine + cisplatin to treat unresectable gastric cancer, and found that grade 3-4 adverse reactions of preoperative treatment reached $69 \%$. Kinoshita et al. [13]. used docetaxel + cisplatin + S-1 to treat unresectable gastric cancer, and reported that grade 3-4 adverse reactions of preoperative treatment reached $31.6 \%$.In contrast, SOX + apatinib has a relatively mild incidence of grade 3-4 adverse reactions.

The surgical conversion rate of this study is $59.5 \%$.The surgical conversion rate reported in the literature is $59.6-73 \%[[3,6,12,13]$. The surgical conversion rate of 
Table 5 Postoperative pathological examination

\begin{tabular}{ll}
\hline Variable & Patients, N (\%) \\
\hline Histologic grade (N=22) & \\
G1. Well differentiated & $0(0)$ \\
G2. Moderately differentiated & $3(13.6)$ \\
G3. Poorly differentiated & $18(64.3)$ \\
Gx. Not evaluated & $1(4.6)$ \\
Pathological response (N=22) & \\
Grade 0 (no effect) & $0(0)$ \\
Grade I (slight effect) & $16(72.7)$ \\
Grade I a (very slight effect) & $7(31.8)$ \\
Grade I b (slight effect) & $9(40.9)$ \\
Grade II (considerable effect) & $5(22.7)$ \\
Grade III (complete response) & $1(4.6)$ \\
\hline
\end{tabular}

this study is not high. The reason is that 9 patients with PR in this study are not willing to accept surgical treatment. Among the patients who underwent R0 resection, one patient achieved pathological complete remission (PCR), which indicated that the scheme had a good conversion effect. No serious complications occurred in any patient during the peri-operative period. The pathological response rate (PRR) reached $68.2 \%$. This standard is widely used in postoperative pathological evaluation of gastric cancer in Asia [14-16]. For the evaluation of primary gastric cancer, the PRR objectively reflects the effectiveness of the combined treatment scheme for patients with advanced gastric cancer.

In terms of survival benefits, the 1-year survival rate of the surgical group was $71.1 \%$, and the 2-year survival rate was $41.1 \%$. The median survival time was 21 months, which was much longer than that of the nonsurgical group in this study, and also longer than patients with advanced gastric cancer who received chemotherapy alone reported in the literature [2], suggesting that the successful conversion and concurrent operation can improve the prognosis of the patients. Kinoshita et al. [13]. used docetaxel + cisplatin + S-1 to treat unresectable gastric cancer, and reported that the 1-, and 2year OS rates were 70.6 and $49.6 \%$, respectively with an MST of 20.9 months. These results suggest that the short-term survival effect of SOX + apatinib in

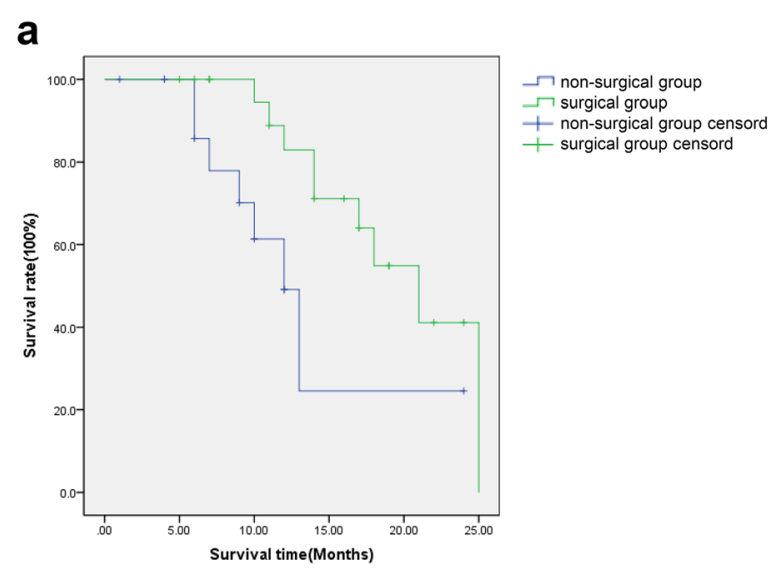

C

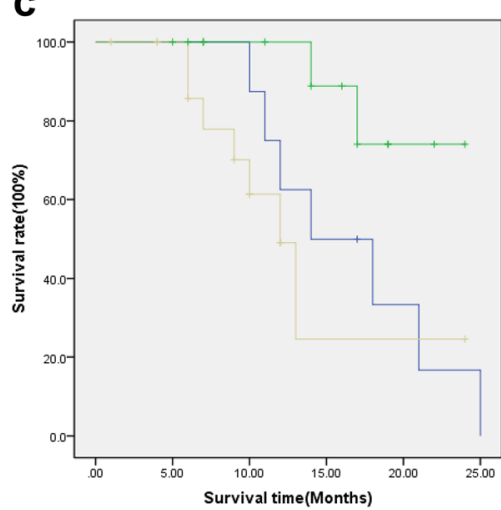

\section{b}

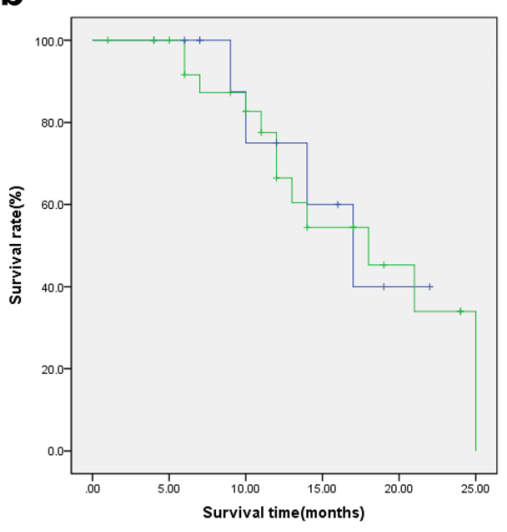

$\neg$ non-peritoneal metastasis $\neg$ peritoneal metastasis + non-peritoneal metastasis-censord + peritoneal metastasis-censord

d

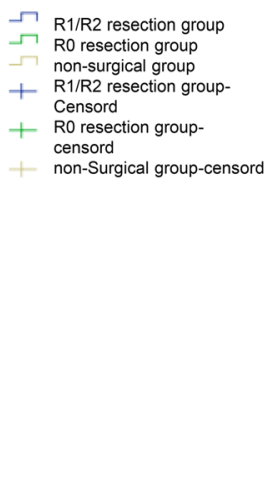

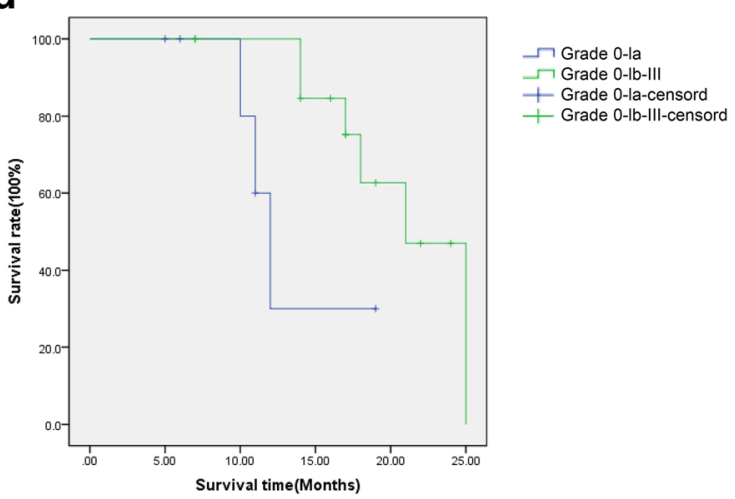

Fig. 3 Shor-term survival effect. a Overall survival curves of the non-surgical and surgical groups; b Overall survival curves of the non-peritoneal metastasis and peritoneal metastasis groups; c Overall survival curves of the R1/R2 resection group, R0 group and non-surgical group; d Overall survival curves of the different pathological responses 
convesion therapy may be close to that of the combination of three chemotherapy drugs. However, there was no significant difference in survival between the peritoneal and non-peritoneal metastasis patients after treatment, suggesting that anti-angiogenesis therapy combined with chemotherapy is also one of the feasible schemes of conversion therapy, even for peritoneal metastasis patients.

The 1-year survival rate and 2-year survival rate of the R0 resection group were 88.9 and $74.1 \%$, respectively, which were similar to the short-term survival of stage III gastric cancer [17-20], which is the ultimate goal of gastric cancer conversion treatment. However, the R1/R2 resection group did not show survival advantage compared with the non-surgical group, which indirectly indicated that if the patients fail to achieve R0 resection after the conversion therapy, surgery may not improve the prognosis. Yamaguchi et al. [21] conducted a multi institution study on 77 patients with stage IV gastric cancer who underwent conversion surgery, and found that the median survival time of patients with R0 was 41.3 months, while that of patients with $\mathrm{R} 1 / 2$ was 21.2 months. This result is similar to that of this study. The 1 -year survival rate of patients who achieved PRR was $84.6 \%$, while the 1-year survival rate of patients who failed to achieve PRR was only $30.0 \%$, which means that the PRR is not only an objective index of efficacy evaluation but also an option for prognosis evaluation.

For a single-arm exploratory clinical study, there are many limitations such as the limited samples size, single research site, and single study population. We will also carry out multi center controlled clinical trials to further verify the feasibility of the scheme.

\section{Conclusions}

In summary, apatinib combined with oxaliplatin and S-1 showed a good short-term survival effect and acceptable safety, especially in patients who were able to undergo R0 resection and achieved grade Ib-III of pathological response after resection.

\section{Abbreviations \\ RO: Radical resection; CR: Complete response; PR: Partial response; SD: Stable disease; PD: Progressive disease; ORR: Objective response rate; DCR: Disease control rate; AEs: Adverse reactions; MDT: Multidisciplinary treatment; SOX: Oxaliplatin and S-1; PRR: Pathological response rate; OSR: Overall survival rate; PCR: Pathological complete remission}

\section{Acknowledgements}

Not applicable.

\section{Authors' contributions}

$Z Y$ and $Y Z$ wrote the main manuscript text and collected data; ZY, ZW and SSC participated in the data analysis; SW, YW, SC and ZL performed the statistical analysis, interpretation of the data, and statistical expertise; ZY and LC carried out the design of the study. All authors reviewed and approved the manuscript.

\section{Funding}

This research was sponsored by the National Clinical Key Specialty Construction Program of China and grants from the National Science Foundation Project of Fujian Science and Technology Department [grant number 2017 J01264, 2018Y0015], Foundation for Fujian Provincial Health Technology Project [grant number 2019-ZQN-16, 2019-CXB-9, 2019006] and Startup Fund for scientific research, Fujian Medical University [grant number 2017Q1219].

\section{Availability of data and materials}

All data generated or analyzed during this study are included in this published article.

\section{Declarations}

Ethics approval and consent to participate

This study was approved by the Institutional Ethics Committee of Fujian Medical University Cancer Hospital (No. 2017-037-03). Written informed consent was obtained from the patients. We confirm that all methods were performed in accordance with the relevant guidelines and regulations by including a statement in the Declaration section.

\section{Consent for publication}

Not applicable.

\section{Competing interests}

The authors declare that they have no competing interests.

\section{Author details}

${ }^{1}$ Department of Gastrointestinal Surgical Oncology, Fujian Cancer Hospital \& Fujian Medical University Cancer Hospital, Fuzhou 350014, Fujian Province, China. 'Department of Fujian Provincial Key Laboratory of Tumor Biotherapy, Fujian Cancer Hospital \& Fujian Medical University Cancer Hospital, Fuzhou 350014, Fujian Province, China.

Received: 13 January 2021 Accepted: 7 June 2021

Published online: 15 June 2021

\section{References}

1. Qiu M, Zhou Y, Zhang X, Wang Z, Wang F, Shao J, et al. Lauren classification combined with HER2 status is a better prognostic factor in Chinese gastric cancer patients. BMC Cancer. 2014;14(1):823. https://doi.org/10.1186/1471-24 07-14-823.

2. Chen $X L$, Chen XZ, Yang C, Liao YB, Li H, Wang L, et al. Docetaxel, cisplatin and fluorouracil (DCF) regimen compared with non-taxane-containing palliative chemotherapy for gastric carcinoma: a systematic review and meta-analysis. PLoS One. 2013;8(4):e60320. https://doi.org/10.1371/journal. pone.0060320.

3. Einama T, Abe H, Shichi S, Matsui H, Kanazawa R, Shibuya K, et al. Longterm survival and prognosis associated with conversion surgery in patients with metastatic gastric cancer. Mol Clin Oncol. 2017;6(2):163-6. https://doi. org/10.3892/mco.2017.1128.

4. Morgagni P, Solaini L, Framarini M, Vittimberga G, Gardini A, Tringali D, et al. Conversion surgery for gastric cancer: a cohort study from a western center. Int J Surg. 2018;53:360-5. https://doi.org/10.1016/j.jjsu.2018.04.016.

5. Saito M, Kiyozaki H, Takata O, Suzuki K, Rikiyama T. Treatment of stage IV gastric cancer with induction chemotherapy using S-1 and cisplatin followed by curative resection in selected patients. World J Surg Oncol. 2014;12(1):406. https://doi.org/10.1186/1477-7819-12-406.

6. Sato Y, Ohnuma H, Nobuoka T, Hirakawa M, Sagawa T, Fujikawa K, et al. Conversion therapy for inoperable advanced gastric cancer patients by docetaxel, cisplatin, and S-1 (DCS) chemotherapy: a multi-institutional retrospective study. Gastric Cancer. 2017;20(3):517-26. https://doi.org/10.1 007/s10120-016-0633-1.

7. Koizumi W, Takiuchi H, Yamada Y, Boku N, Fuse N, Muro K, et al. Phase II study of oxaliplatin plus $\mathrm{S}-1$ as first-line treatment for advanced gastric cancer (G-SOX study). Ann Oncol. 2010;21(5):1001-5. https://doi.org/10.1 093/annonc/mdp464.

8. Kim GM, Jeung HC, Rha SY, Kim HS, Jung I, Nam BH, et al. A randomized phase II trial of S-1-oxaliplatin versus capecitabine-oxaliplatin in advanced 
gastric cancer. Eur J Cancer. 2012;48(4):518-26. https://doi.org/10.1016/j. ejca.2011.12.017.

9. Yamada Y, Higuchi K, Nishikawa K, Gotoh M, Fuse N, Sugimoto N, et al. Phase III study comparing oxaliplatin plus S-1 with cisplatin plus S-1 in chemotherapy-naive patients with advanced gastric cancer. Ann Oncol. 2015;26(1):141-8. https://doi.org/10.1093/annonc/mdu472.

10. Li J, Qin S, Xu J, Xiong J, Wu C, Bai Y, et al. Randomized, double-blind, placebo-controlled phase III trial of Apatinib in patients with chemotherapyrefractory advanced or metastatic adenocarcinoma of the stomach or gastroesophageal junction. J Clin Oncol. 2016;34(13):1448-54. https://doi. org/10.1200/JCO.2015.63.5995.

11. Zheng Y, Yang X, Yan C, Feng R, Sah BK, Yang Z, et al. Effect of apatinib plus neoadjuvant chemotherapy followed by resection on pathologic response in patients with locally advanced gastric adenocarcinoma: a single-arm, open-label, phase II trial. Eur J Cancer. 2020;130:12-9. https://doi. org/10.1016/j.ejca.2020.02.013.

12. Sym SJ, Chang HM, Ryu MH, Lee JL, Kim TW, Yook JH, et al. Neoadjuvant docetaxel, capecitabine and cisplatin (DXP) in patients with unresectable locally advanced or metastatic gastric cancer. Ann Surg Oncol. 2010;17(4): 1024-32. https://doi.org/10.1245/s10434-009-0838-1.

13. Kinoshita J, Fushida S, Tsukada T, Oyama K, Okamoto K, Makino I, et al. Efficacy of conversion gastrectomy following docetaxel, cisplatin, and S-1 therapy in potentially resectable stage IV gastric cancer. Eur J Surg Oncol. 2015:41(10):1354-60. https://doi.org/10.1016/j.ejso.2015.04.021.

14. Hirakawa M, Sato Y, Ohnuma H, Takayama T, Sagawa T, Nobuoka T, et al. A phase II study of neoadjuvant combination chemotherapy with docetaxel, cisplatin, and S-1 for locally advanced resectable gastric cancer: nucleotide excision repair (NER) as potential chemoresistance marker. Cancer Chemother Pharmacol. 2013;71(3):789-97. https://doi.org/10.1007/s00280013-2073-5.

15. Yoshikawa T, Omura K, Kobayashi O, Nashimoto A, Takabayashi A, Yamada T, et al. A phase II study of preoperative chemotherapy with S-1 plus cisplatin followed by D2/D3 gastrectomy for clinically serosa-positive gastric cancer (JACCRO GC-01 study). Eur J Surg Oncol. 2010;36(6):546-51. https:// doi.org/10.1016/j.ejso.2010.04.011.

16. Yoshikawa T, Sasako M, Yamamoto S, Sano T, Imamura H, Fujitani K, et al. Phase II study of neoadjuvant chemotherapy and extended surgery for locally advanced gastric cancer. Br J Surg. 2009;96(9):1015-22. https://doi. org/10.1002/bjs.6665.

17. Suh SO, Kroh M, Kim NR, Joh YG, Cho MY. Effects of red ginseng upon postoperative immunity and survival in patients with stage III gastric cancer. Am J Chin Med. 2002;30(4):483-94. https://doi.org/10.1142/S0192415X02 000661.

18. Takahari D, Hamaguchi T, Yoshimura K, Katai H, Ito S, Fuse N, et al. Survival analysis of adjuvant chemotherapy with $\mathrm{S}-1$ plus cisplatin for stage III gastric cancer. Gastric Cancer. 2014;17(2):383-6. https://doi.org/10.1007/s10120-0130264-8.

19. Shu P, Qin J, Shen K, Chen W, Liu F, Fang Y, et al. The IGCA staging system is more accurate than AJCC7 system in stratifying survival of patients with gastric cancer in stage III. BMC Cancer. 2017:17(1):238. https://doi.org/10.11 86/s12885-017-3235-3.

20. Ito $\mathrm{Y}$, Kanda $\mathrm{M}$, Ito $\mathrm{S}$, Mochizuki $\mathrm{Y}$, Teramoto $\mathrm{H}$, Ishigure $\mathrm{K}$, et al. Intraoperative blood loss is associated with shortened postoperative survival of patients with stage II/III gastric Cancer: analysis of a multi-institutional dataset. World J Surg. 2019;43(3):870-7. https://doi.org/10.1007/s00268-01 8-4834-0.

21. Yamaguchi K, Yoshida K, Tanahashi T, Takahashi T, Matsuhashi N, Tanaka Y, et al. The long-term survival of stage IV gastric cancer patients with conversion therapy. Gastric Cancer. 2018;21(2):315-23. https://doi.org/10.1 007/s10120-017-0738-1.

\section{Publisher's Note}

Springer Nature remains neutral with regard to jurisdictional claims in published maps and institutional affiliations.

Ready to submit your research? Choose BMC and benefit from:

- fast, convenient online submission

- thorough peer review by experienced researchers in your field

- rapid publication on acceptance

- support for research data, including large and complex data types

- gold Open Access which fosters wider collaboration and increased citations

- maximum visibility for your research: over $100 \mathrm{M}$ website views per year

At $\mathrm{BMC}$, research is always in progress.

Learn more biomedcentral.com/submissions 